\title{
Filament simulations in regions of highly-varying parallel connection length
}

\author{
B. Shanahan ${ }^{\circledR 1, \dagger}$ and P. Huslage ${ }^{\circledR 2}$ \\ ${ }^{1}$ Max-Planck-Institut für Plasmaphysik, Wendelsteinstr. 1, 17491 Greifswald, Deutschland \\ ${ }^{2}$ Fakultät für Physik und Astronomie, Am Hubland, 97074 Würzburg, Deutschland
}

(Received 20 February 2020; revised 15 May 2020; accepted 18 May 2020)

The island divertor topology of the Wendelstein 7-X (W7-X) scrape-off-layer exhibits regions of highly varying connection length. Here, we present drift-plane simulations of seeded filaments in regions of sharp transitions in parallel connection length - a parameter which dictates the propagation regime for plasma blobs. It is determined that a transition in parallel connection length alters the trajectory of filaments; filaments which enter regions of lower connection length are decelerated, and vice versa. It is also determined that if the lobes of a potential dipole created by diamagnetic drifts within the filament exist in two regions of distinct parallel connection length, the filament is then steered towards the region of higher connection length. The extreme case of a narrow region of varying connection length can also alter the trajectory of a filament, depending on the extent of this region. Finally, simulations mimicking the view from the W7-X gas puff imaging (GPI) diagnostic view plane are presented. It is determined that filaments in the view of the W7-X GPI diagnostic exhibit a predominantly poloidal propagation due to the radial electric field, since the radial velocity is relatively small.

Key words: plasma nonlinear phenomena, plasma sheaths, plasma simulation

\section{Introduction}

Large, coherent, field-aligned filaments - or blobs - are a major source of particle transport in the scrape off layer (SOL) of tokamaks. These filaments are primarily advected by $E \times B$ effects due to charge separation arising from diamagnetic drifts, where $E$ and $B$ are the internal binormal electric field within the filament and toroidal magnetic field, respectively. The resulting transport is determined by the mechanism mitigating the charge separation. Blobs with a short connection length $\left(L_{\|}\right)$to the sheath resolve charge separation via parallel currents, but if the connection length is long enough (or the blob small enough) the charge can be short-circuited via perpendicular currents. Other factors such as magnetic shear and flux expansion can also affect filament motion, but are neglected in this work. A very thorough discussion of filament propagation can be found in D'Ippolito, Myra \& Zweben (2011).

$\dagger$ Email address for correspondence: brendan.shanahan@ipp.mpg.de 
The recent experimental campaigns on Wendelstein 7-X have exhibited evidence of filaments advecting in the edge and SOL (Kocsis et al. 2017; Zoletnik et al. 2019; Killer et al. 2020). The edge and SOL topology of the Wendelstein 7-X stellarator provides a novel environment for filament physics. Unlike tokamak scrape-off-layers, W7-X exhibits abrupt transitions in connection length (Hammond et al. 2019) due to the edge island topology.

Filament physics in stellarators is only starting to be investigated numerically, primarily due to the difficulties incorporating the complex geometry. The BSTING project (Shanahan, Dudson \& Hill 2019) has provided a framework for global edge and SOL turbulence simulations in Wendelstein 7-X, but filament physics often benefits from simplifications. Simulations of blobs in slab geometries are common (D'Ippolito \& Myra 2003; D'Ippolito et al. 2004; Aydemir 2005; Garcia et al. 2005; Garcia, Bian \& Fundamenski 2006; Myra, Russell \& D’Ippolito 2006; Riva et al. 2016; Shanahan \& Dudson 2016; Walkden et al. 2016) since these simulations are much faster than global edge simulations, allowing thorough investigations of the primary mechanisms in filament dynamics. This method has already been applied to non-uniform drive scenarios (Shanahan, Dudson \& Hill 2018), a feature which is prominent in - but not exclusive to - stellarators. In Shanahan et al. (2018) it was determined that a filament which is driven by non-uniform curvature will, on average, propagate as if driven by the field-line-averaged curvature - except in cases of highly non-uniform drive. Additionally, it was determined by Killer et al. (2020) that simulations in drift-plane geometries effectively recover the macroscopic transport dynamics of filaments measured in experiment. Here we note these results in order to motivate simulation of filaments in drift plane geometries with abrupt parallel connection length transitions. The results presented in this manuscript are the first attempt at understanding the effects of abrupt $L_{\|}$transitions - like those found in the island divertor region of $\mathrm{W} 7-\mathrm{X}$ - on filament transport.

Section 2 uses an isothermal model to investigate the motion of filaments near abrupt transitions in $L_{\|}$. Section 3 introduces a non-isothermal model and discusses simulation results which mimic the $L_{\|}$profile as viewed by the gas puff imaging (GPI) diagnostic on Wendelstein 7-X. A summary of the results and discussion of the implications for filamentary transport in $\mathrm{W} 7-\mathrm{X}$ is presented in $\S 4$.

\section{Simulations in drift-plane geometry}

In this work, the effects of $L_{\|}$transitions are investigated using two different, two-dimensional fluid turbulence models implemented in the BOUT++ (Dudson et al. 2009, 2016, 2019) framework. Each model utilizes parallel closure terms to include the parallel connection length to the plasma facing components. The isothermal model presented in $\$ 2.1$ (blob2d) is a well-established model for investigating filament physics, and therefore provides a reliable test bed for studying the effects of $L_{\|}$transitions. The second model, Hermes, is a full-profile, non-isothermal model with flux-conservative operators which is still in active development. This work represents the first use of the Hermes model for filament dynamics in drift-plane geometries. Therefore, to facilitate understanding of the underlying mechanisms, the following sections will utilize blob2d to determine what effects $L_{\|}$transitions have on filaments and the more sophisticated Hermes model will be used in $\S 3$ to provide insight into experimental scenarios. 


\subsection{Isothermal model}

First, we use the blob2d example provided in the BOUT++ open-source repository (Dudson et al. 2019) to perform seeded filament simulations in regions of varying connection length. This model is a two-dimensional version of the model in Walkden, Dudson \& Fishpool (2013), and is a very common model for filament simulations (Benkadda, Garbet \& Verga 1994; Yu \& Krasheninnikov 2003; D’Ippolito et al. 2004; Myra et al. 2004; Aydemir 2005; Garcia et al. 2006) which evolves plasma density $n$ and vorticity $\omega$ in SI units

$$
\begin{gathered}
\frac{\mathrm{d} n}{\mathrm{~d} t}=2 c_{s} \rho_{s} \xi \cdot(\nabla n-n \nabla \phi)+\frac{n \phi}{L_{\|}}, \\
\rho_{s}^{2} n \frac{\mathrm{d} \omega}{\mathrm{d} t}=2 c_{s} \rho_{s} \xi \cdot \nabla n+\frac{n \phi}{L_{\|}},
\end{gathered}
$$

where $c_{s}$ is the sound speed and $\rho_{s}$ is the gyroradius. Total derivatives are split via $\mathrm{d} / \mathrm{d} t=\partial / \partial t+\boldsymbol{u}_{E} \cdot \nabla$ where $\boldsymbol{u}_{E}=\boldsymbol{b} \times \nabla \phi / B$ is the $E \times B$ velocity. The vorticity is given by $\omega \equiv n / B \nabla_{\perp}^{2} \phi$, where the Boussinesq approximation has been used (Yu \& Krasheninnikov 2003; D'Ippolito et al. 2004; Angus \& Umansky 2014; Wiesenberger, Madsen \& Kendl 2014). The reference sound speed is $c_{s 0}=\sqrt{T_{e 0} / m_{i}}$. Density is normalized to a reference density $n_{0}$ and the electrostatic potential $\phi$ is normalized to the background (electron) temperature $T_{e 0}(\mathrm{eV})$, such that $\phi=e \Phi / T_{e 0}$ where $\Phi$ is the plasma potential. Curvature is implemented via $\xi=(\nabla \times b / B) \approx 2 / B \boldsymbol{b} \times \kappa \approx 1 / R_{c}$ where $R_{c}$ is the radius of curvature. In this model the closure terms - the final terms on the right-hand side - are provided from the sheath dissipation model (Garbet et al. 1991; Krasheninnikov 2001; D'Ippolito, Myra \& Krasheninnikov 2002; D'Ippolito \& Myra 2003; D'Ippolito et al. 2004; Krasheninnikov, Ryutov \& Yu 2004; Garcia et al. 2006; Myra et al. 2006; Kube \& Garcia 2011; Easy et al. 2014; Schwörer et al. 2018), which is an approximation for the parallel current gradient $\left(1 / e \nabla_{\|} J_{\|}\right)$ and provides an $L_{\|}$dependence for parallel sheath closure.

Unless otherwise stated, filaments in this paper are initialized as a $100 \%$ Gaussian perturbation above the background density - the peak density $n_{\text {peak }}$ is double the background density, $n_{\text {peak }}=2 n_{0}$. The background parameters were chosen to be W7-X-relevant: $T_{e 0}=100 \mathrm{eV}, n_{0}=2 \times 10^{19} \mathrm{~m}^{-3}, R=6 \mathrm{~m}, B_{0}=2.5 \mathrm{~T}$. In the $L_{\|}$regimes discussed here, these parameters result in a dimensionless collisionality (Editors et al. 1999) of $v^{*} \approx 2$ for $L_{\|}=10 \mathrm{~m}$, and $v^{*} \approx 200$ for $L_{\|}=1 \mathrm{~km}$, which is an appropriate regime for fluid simulations where it is often assumed $v^{*} \gg 1$. Furthermore, if using the collisionality parameter from (for instance) Myra et al. (2006), we arrive at $\Lambda \approx 0.3$ for $L_{\|}=10 \mathrm{~m}$, which is well within the range for fluid simulations. An example of the initial conditions for the following simulations are shown in figure 1.

The simulations in this section are performed on a $512 \times 512$ grid with a resolution of $2 \rho_{s} \approx 1 \mathrm{~mm}$. Zero-gradient (Neumann) boundary conditions have been applied on all fields. Laplacian inversion was implemented using the PETSc Balay et al. (1997) solver within BOUT++. All models and inputs within this work are, like BOUT++, open source and freely available (see acknowledgements).

This paper will be devoted by examining the affects of $L_{\|}$transitions on blob propagation. The following subsection first determines the effect that parallel connection length has on the scaling of filament velocity with perpendicular size, which will help to understand the underlying physics at a $L_{\|}$transition. 


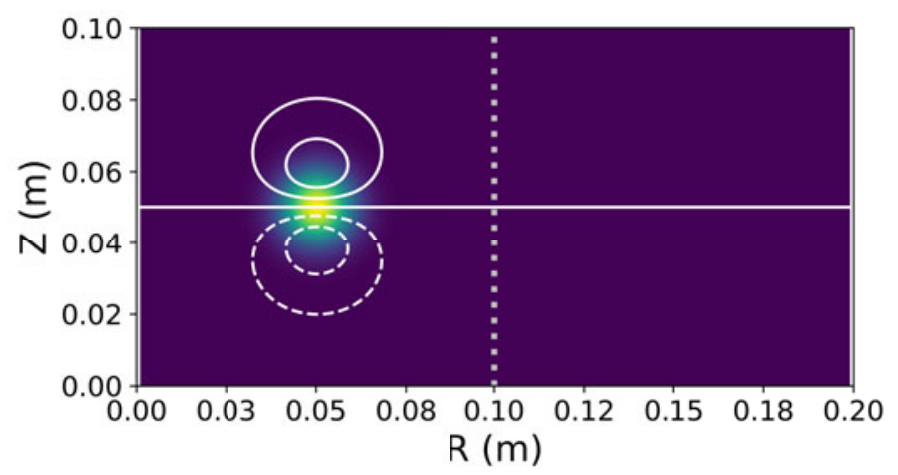

FIGURE 1. Initial conditions of a filament near a transition. The density is initialized as a circular Gaussian (colour contour). The potential dipole (white contour) is allowed to develop before encountering a transition in $L_{\|}$(dashed vertical line).

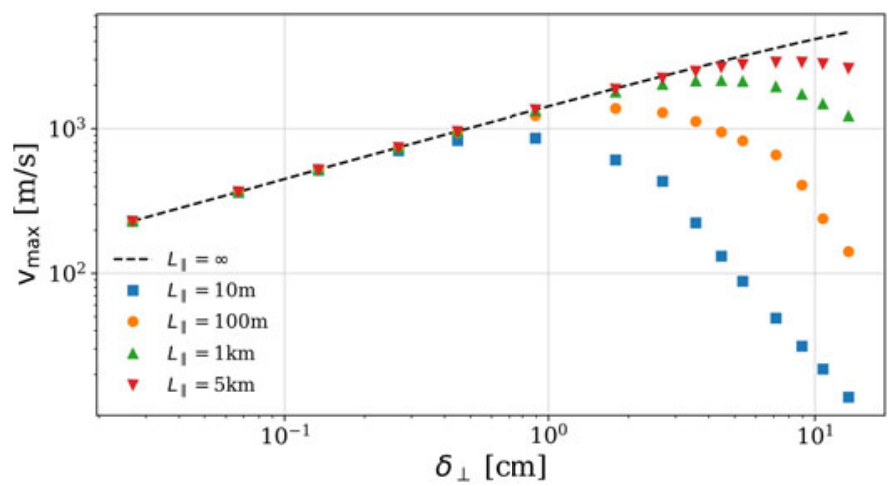

FIGURE 2. Filament velocity scaling in various connection length regimes. Sheath effects become apparent for smaller filaments at shorter connection lengths.

\subsection{Blob scaling in different connection length regimes}

A filament where the current is dissipated through the sheath is considered to propagate in the sheath-limited regime. In this regime, the velocity of the filament scales as $\delta_{\perp}^{-2}$, where $\delta_{\perp}$ is the perpendicular blob size. If, however, a filament is in closed-field-line regimes, it scales as $\delta_{\perp}^{1 / 2}$, and is said to be inertially limited. A thorough discussion of these regimes, and derivations of the scaling with perpendicular size can be found in Krasheninnikov (2001) and D'Ippolito et al. (2011). Figure 2 illustrates the scaling of filament velocity versus $\delta_{\perp}$ for various connection lengths as simulated using the blob2d model.

From figure 2 it is noted that even filaments with very long connection lengths will exhibit a shoulder into the sheath-limited regime, although this effect is most prominent for very large filaments. Unless otherwise stated, filaments in the following sections will be initialized at approximately the 'fundamental blob size' (D'Ippolito et al. 2011), which generally lies at the crossing point of inertially and sheath-limited scalings - which leads to highly coherent motion. As is evident in figure 2, this depends on the parallel connection length. An expression for which can be found 


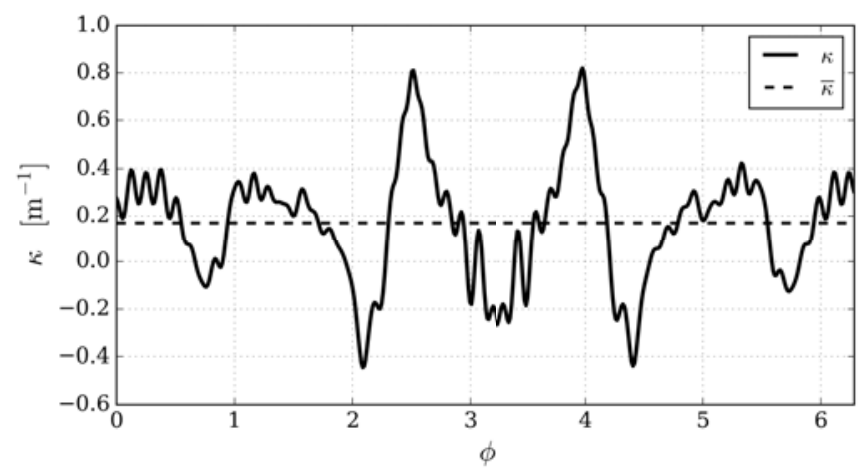

FIGURE 3. Field-line curvature of a field line at the centre of the GPI view (see $\S 3$ ) in the SOL of W7-X. Field-line curvature is highly non-uniform, but strong deviations from the mean are toroidally localized.

in Yu \& Krasheninnikov (2003) as

$$
\delta_{*}=2 \rho_{s}\left(\frac{L_{\|}}{\rho_{s}}\right)^{2 / 5} \eta_{\mathrm{pol}}^{1 / 5},
$$

where $\eta_{\text {pol }}$ describes the plasma polarizing mechanism, which depends on the magnetic configuration. In linear devices, Yu \& Krasheninnikov (2003) assert that the plasma is polarized due to centrifugal and neutral wind effects, so $\eta_{\text {pol }}$ is a function of the neutral velocity and ion-neutral collision frequency. In tokamaks, it is often assumed that the polarization mechanism is due predominantly to the toroidal magnetic field, thus $\eta_{\text {pol }} \approx 2 \rho_{s} / R$.

If, instead, we substitute the local field-line curvature for a field line in the scrape-off-layer of Wendelstein $7-X$ for the polarization mechanism, we find that the expected blob size will vary anywhere between 1 and $3 \mathrm{~cm}$ due to the local field-line curvature. Thus, noting that connection lengths in W7-X are between 10 and several hundreds of meters, we refer to figure 2 and note that filaments can span the transition from inertially to sheath-limited propagation regimes. It is therefore difficult to determine the filament size which will lead to the most coherent propagation in Wendelstein 7-X. Experimental investigation of scrape-off-layer filaments in Wendelstein 7-X is still quite novel (Zoletnik et al. 2019; Killer et al. 2020), so a detailed analysis of coherent filament propagation in this geometry is lacking.

However, a reasonable value for the initial filament diameter must be chosen. Referring to figure 3, we see that the field-line curvature exhibits only narrow peaks along the field line. As such, the field-line curvature only briefly deviates far from the average value. Using this knowledge, and acknowledging the work in Shanahan et al. (2018), we therefore use the $\delta_{*}$ value corresponding to the average curvature drive, which is approximately $2 \mathrm{~cm}$.

\subsection{Vertical transitions}

The scrape-off-layer of Wendelstein 7-X exhibits a patchwork $L_{\|}$structure, where neighbouring field lines can have completely different connection lengths - by over an order of magnitude (Hammond et al. 2019). For the following simulations, 


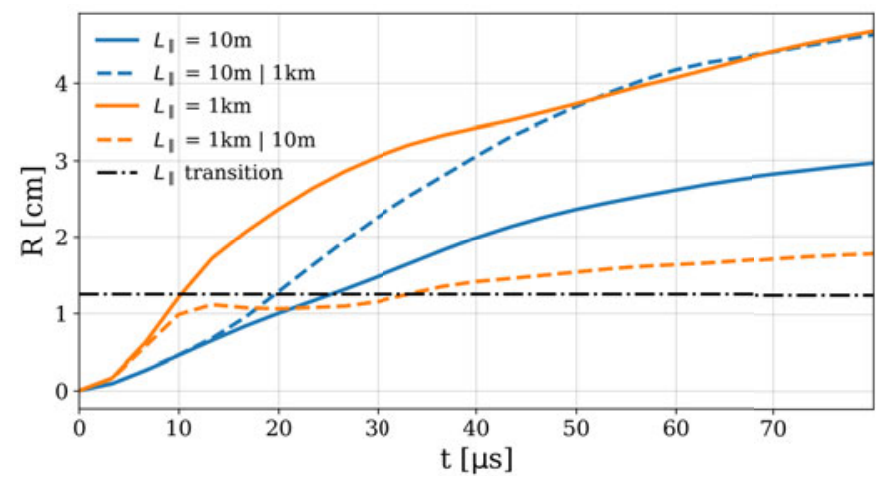

FIGURE 4. The effect of an $L_{\|}$transition; a filament entering a region of lower connection length (orange, dashed) will be decelerated relative to the constant- $L_{\|}$case (orange, solid). In the opposite case, a filament is accelerated (blue, dashed) relative to the case with constant $L_{\|}$(blue, solid).

a connection length transition was placed perpendicular to the propagation of seeded filaments. Figure 4 indicates the effect of a transition by plotting the radial displacement a filament's centre of mass $\left(\delta_{\perp} \approx 1.7 \mathrm{~cm}\right)$ versus time.

The trajectory of a filament is altered when it crosses a transition in parallel connection length. A filament entering a different connection length regime will jump to the respective propagation curve from figure 2 - blobs which enter regions of shorter connection length are decelerated relative to the case without a transition, while the opposite transition causes an acceleration. One surprising result is that a filament which transitions from a $10 \mathrm{~m}$ region into a $1 \mathrm{~km}$ region travels farther than a filament which encounters no transition, and remains in a $1 \mathrm{~km}$ region. This indicates that the blob is coherent enough by the time it encounters the transition to act as if it were seeded in a region of $1 \mathrm{~km}$ connection length. This effect is not apparent if the transition is placed further away from the initial seeding location. In such simulations where the initial seeding location is farther from the transition, the blob loses coherence before reaching the transition and the effects of the transition are reduced.

This deceleration can be exaggerated in larger filaments. A large filament which is near the fundamental blob size for a $1 \mathrm{~km}$ connection length (approximately $7 \mathrm{~cm}$ ) will enter the $10 \mathrm{~m}$ region and immediately have its charge dissipated, and is therefore almost entirely blocked at the transition location (figure 5). Secondary filaments are then generated which propagate slowly into the $10 \mathrm{~m}$ region.

\subsection{The effects of transition magnitude}

Having established that the filament trajectory is altered by a transition in $L_{\|}$, it is natural to investigate if the strength of the transition - how large the step in $L_{\|}$is - drastically affects the blob propagation. Figure 6 indicates the radial position of an approximately $2 \mathrm{~cm}$ filament as a function of time in cases with varying transition strength.

From figure 6 it appears that even small transitions (by W7-X standards) significantly alter propagation. Therefore, it could be possible to measure the altered filamentary propagation near regions of highly varying connection length, as a deceleration is seen in even in moderate-amplitude transitions. 

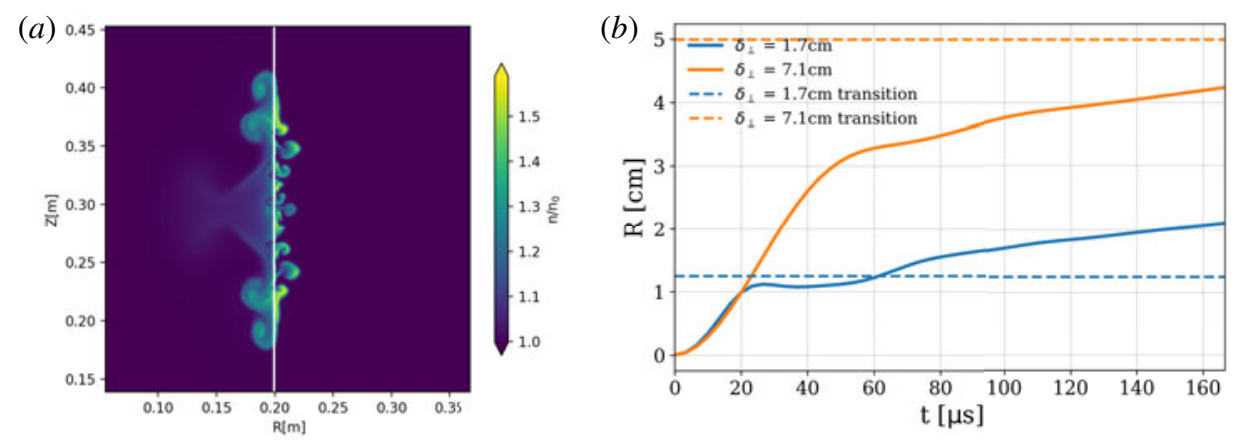

FIgURE 5. (a) A filament encountering a transition to a lower connection length can, in some cases, be strongly decelerated, generating secondary filaments beyond the transition. (b) The effect of the $L_{\|}$transition is more apparent for larger filaments (orange lines), where sheath effects are more prominent.

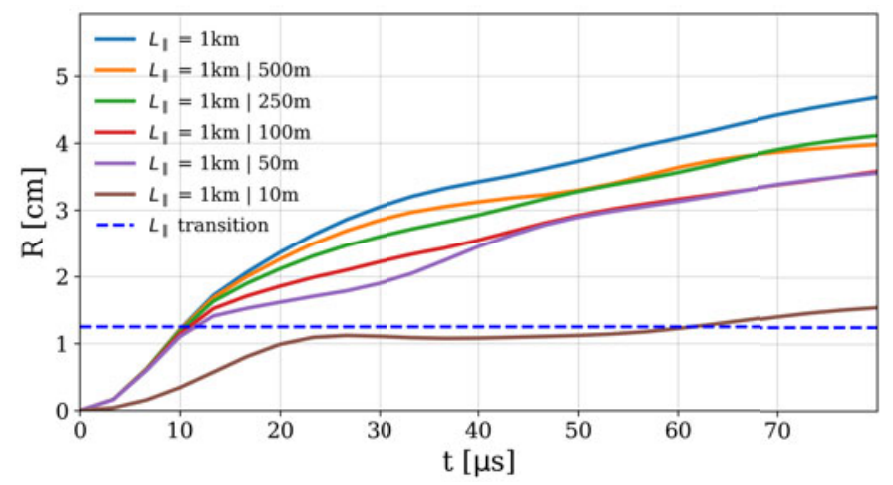

FIGURE 6. The effect of the transition magnitude; filaments are decelerated when entering regions of lower connection length, and this effect scales monotonically with the magnitude of the transition.

\subsection{Horizontal transition}

A filament will not always encounter a transition which is perpendicular to the propagation trajectory. For this reason, the case of a filament where the two lobes of the potential dipole (which develop due to diamagnetic drifts) evolves in two distinct regions of $L_{\|}$was considered. In the geometry shown in figure 1, the transition would be horizontal, bisecting the filament. Figure 7 indicates the maximum vertical displacement of for different initial filament diameters, where the filaments are initialized across a transition in $L_{\|}$from $10 \mathrm{~m}$ to $1000 \mathrm{~m}$, where $z=0$ is the position of the transition.

Filaments whose potential dipole evolves in two separate connection length regimes are steered into the region of higher connection length. At higher connection lengths, parallel currents cannot sufficiently mitigate the charge separation, and the resulting vorticity steers the filament into this region. Maximum vertical displacement initially increases with filament size, but filaments with sufficient size (in this case, $\delta_{\perp}=5 \mathrm{~cm}$ ) will be sheared apart, which spawns secondary filaments which independently evolve. The filaments in smaller connection length regimes exhibit a negligible velocity 


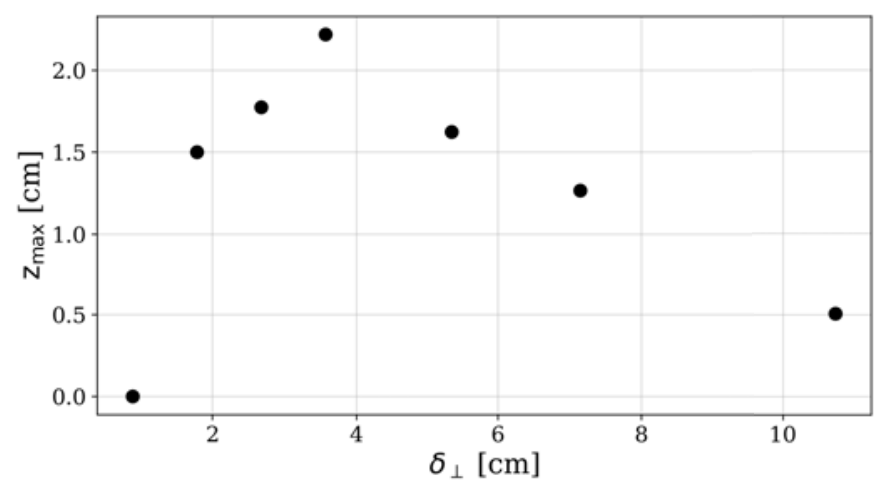

FIGURE 7. Scaling of maximum normal displacement of a filament whose dipole develops in two regions of distinct $L_{\|}$. Filaments are steered more strongly into the region of higher connection length, until the sheering is strong enough to lose coherence (here shown around $\delta_{\perp} \approx 5 \mathrm{~cm}$ ), and secondary filaments are advected independently.

compared to those in $L_{\|}=1 \mathrm{~km}$ regions. The net result is that the centre of mass does not stray far from the origin, as shown for larger filament diameters in figure 7.

\subsection{Thin areas of varying $L_{\|}$}

A filament can encounter multiple transitions in $L_{\|}$before it is dissipated. We investigate here how the distance between two transitions can affect the filament trajectory. First, we investigate how a filament propagates when first encountering a transition to higher connection length $(1 \mathrm{~km})$, followed by another step back down to $10 \mathrm{~m}$. This case has an analogue in the W7-X SOL, where the separatrix in island diverted scenarios exhibits very high connection lengths, which are adjacent to areas of short connection length. In this example, an approximately $2 \mathrm{~cm}$ Gaussian filament encounters regions which are one fourth its standard deviation $(\sigma / 4)$ and 4 times its standard deviation $(4 \sigma)$ in width - shown as shaded regions in figure 8 .

Figure 8 indicates that a filament encountering a small region of increased parallel connection length will be accelerated relative to the case without a transition, but if that region is large enough, the deceleration at the second transition will dictate that the filament does not propagate as far. Figure 9 illustrates the opposite case: an approximately $2 \mathrm{~cm}$ filament which encounters a region of lower $L_{\|}$, with regions which are less than or equal to its standard deviation $(\sigma)$ in width.

A filament briefly encountering a region of shorter connection length will be decelerated, even in cases of very narrow regions of smaller $L_{\|}$. Although the filament dipole exists only briefly in the region of short connection length, the parallel currents are able to sufficiently dissipate the charge separation, leading to drastically reduced transport.

\section{Filament transport in the W7-X GPI field of view}

Wendelstein 7-X will have a GPI diagnostic installed for the second operational phase, allowing for visual tracking of plasma filaments in the edge and scrape off layer. The exact regions of the edge and scrape off layer within viewed by the GPI diagnostic depends on the magnetic configuration. Figure 10 indicates the connection lengths for the standard magnetic configuration ('EIM') in Wendelstein 7-X. 


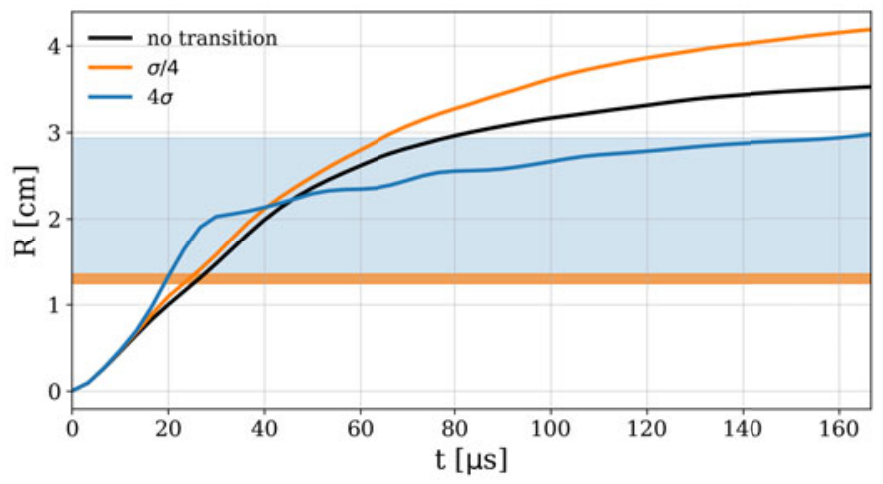

FIGURE 8. Filament trajectory (lines) when encountering limited areas of higher $L_{\|}$(shaded regions). A small area of increased $L_{\|}$(orange) - which is one fourth of the filament standard deviation in width - can accelerate a filament, but if the second transition is far enough - in this case, four filament standard deviations from the first transition - from the first (blue), it can have a net decelerating effect.

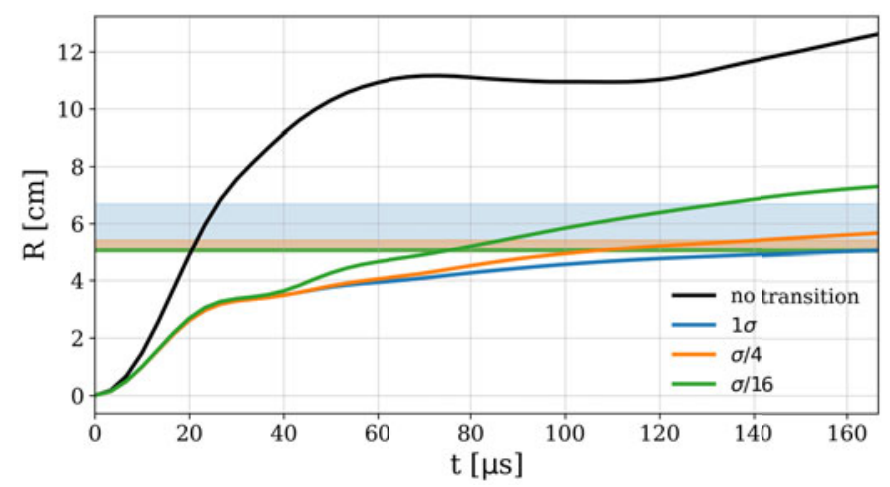

FIGURE 9. A small area of low parallel connection length (shaded regions) causes a significant deceleration in the filament trajectory (lines). This holds even if this section is much smaller than the size of the initial Gaussian perturbation.

Figure 10 indicates that the GPI diagnostic could witness filaments encountering connection length transitions. Here, we use a non-isothermal model to seed filaments in these scenarios with initial conditions which mirror W7-X experimental parameters.

\subsection{Hermes, a non-isothermal model}

Simulations performed in this section utilize the Hermes model (Dudson \& Leddy 2017), which is a non-isothermal, cold-ion, full-profile model. This model introduces the added physics of (among other effects) electron pressure which was missing in the previous section using blob2d. The inclusion electron pressure in filament simulations has been explored, for instance, in Walkden et al. (2016). We do not expect drastic changes to filament behaviour following the inclusion of electron pressure as we are initializing the filaments with a flat temperature background. Therefore, while the filaments are initialized as isothermal, the temperature is free to fluctuate. The system of equations used here is solved only in the drift plane, and therefore parallel effects 


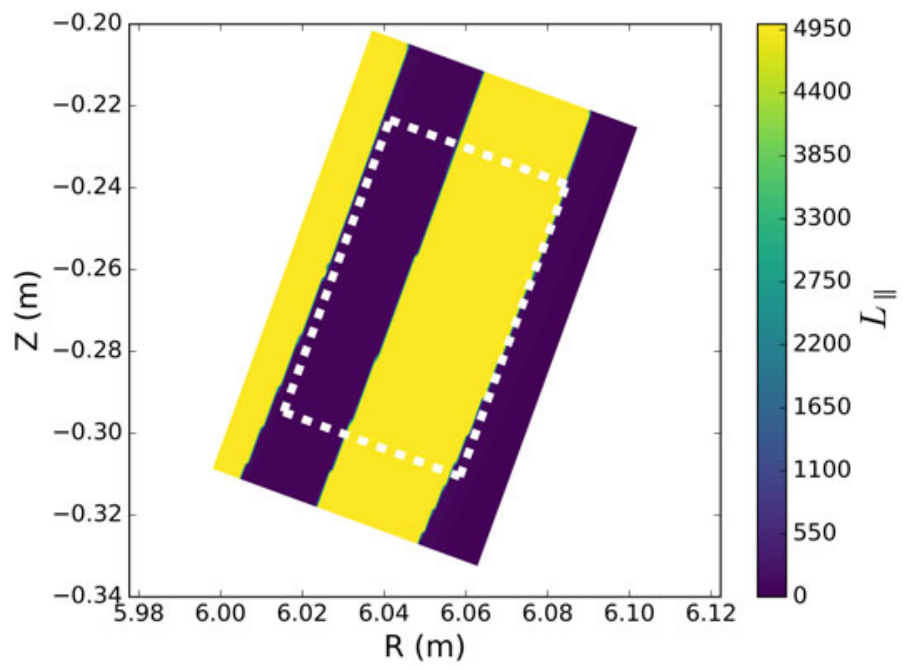

FIGURE 10. Parallel connection length regimes as viewed by the GPI diagnostic in the W7-X standard magnetic configuration. The GPI diagnostic will view open field lines in the SOL with $L_{\|} \approx 50 \mathrm{~m}$ (left), and the island O-point region with closed field lines (right).

are only included in the closure terms. Therefore the equations which evolve density $n$, electron pressure $p_{e}$, and vorticity $\omega$ in SI units are as follows:

$$
\begin{gathered}
\frac{\partial n}{\partial t}=-\nabla \cdot\left(n \boldsymbol{V}_{E \times B}+n \boldsymbol{V}_{\mathrm{mag}}\right)-\frac{\sqrt{T_{i 0} / m_{i}}}{2 L_{\|}} n, \\
\frac{3}{2} \frac{\partial p_{e}}{\partial t}=-\nabla \cdot\left(\frac{3}{2} p_{e} \boldsymbol{V}_{E \times B}+p_{e} \frac{5}{2} \boldsymbol{V}_{\mathrm{mag}}\right)-p_{e} \boldsymbol{\nabla} \cdot \boldsymbol{V}_{E \times B}-T_{e} \frac{\sqrt{T_{i 0} / m_{i}}}{2 L_{\|}} n, \\
\frac{\partial \omega}{\partial t}=-\nabla \cdot\left[\frac{1}{2}\left(\omega+\frac{e n}{\Omega_{i} B} \nabla_{\perp}^{2} \phi\right) \boldsymbol{V}_{E \times B}\right]+\nabla_{\perp} \cdot\left(\frac{1}{2} \frac{\partial n_{i}}{\partial t} \frac{e}{\Omega_{i} B} \nabla_{\perp} \phi\right) \\
-\nabla \cdot\left(e n \boldsymbol{V}_{\mathrm{mag}}\right)+\nabla \cdot\left(\mu_{i} \nabla_{\perp} \omega\right)+\frac{e n c_{s}}{L_{\|}} \sqrt{T_{e}}\left[1-\sqrt{\frac{m_{i}}{4 \pi m_{e}}} \exp \left(-e \phi / T_{e}\right)\right],
\end{gathered}
$$

where density is normalized to a reference density $n_{0}$, temperature and electrostatic potential $\phi$ to a reference $T_{0}$ (in $\mathrm{eV}$ ) and pressure is normalized to $p_{0}=n_{0} T_{0}$. The reference sound speed is given by $c_{s 0}=\sqrt{T_{0} / m_{i}}$. The ion viscosity coefficient $\mu_{i}$ is defined in Madsen (2013).

The $E \times B$ and magnetic drifts are given by the drift velocities

$$
\boldsymbol{V}_{E \times B}=\frac{\boldsymbol{b} \times \nabla \phi}{B}, \quad \boldsymbol{V}_{\mathrm{mag}}=-\frac{T_{e}}{e} \nabla \times \frac{\boldsymbol{b}}{B} .
$$

All simulations presented here are performed without the Boussinesq approximation (although this is an option in Hermes), and therefore the vorticity is given by

$$
\omega=\nabla \cdot\left(\frac{e n}{\Omega_{i} B} \nabla_{\perp} \phi\right) .
$$


As the plasma model is cast in conservative form, it conserves particle number and an energy (when neglecting the parallel closure terms)

$$
E=\int \mathrm{d} v\left(\frac{1}{2} \frac{n e^{2}}{\Omega_{i}}|\nabla \phi|^{2}+\frac{3}{2} p_{e}\right),
$$

where the terms correspond to the ion $E \times B$ energy and electron thermal energy. Differential operators are discretized using flux-conservative finite volume methods, which are discussed in Dudson \& Leddy (2017).

The final terms in (3.1)-(3.3) are parallel closure terms, which are approximations for the parallel sheath dynamics and break the aforementioned energy conservation. The closures in Hermes differ from those in blob2d in that they assume that the ion flow dominates over the parallel current to the sheath, as is evident from the dependence on the ion temperature $T_{i 0}$ which is set here to be the same as the normalization temperature $T_{0}$ (although this is not required in Hermes). The sheath dissipation model used in blob2d (Garbet et al. 1991; Krasheninnikov 2001; D'Ippolito et al. 2002; D'Ippolito \& Myra 2003; D'Ippolito et al. 2004; Krasheninnikov et al. 2004; Garcia et al. 2006; Myra et al. 2006; Kube \& Garcia 2011; Easy et al. 2014; Schwörer et al. 2018) includes only electron parallel motion, assuming the parallel ion flow is small. Nevertheless, the results of Hermes simulations in the scenarios presented in $\S 2$ exhibit the same phenomena as previously presented with blob2d. In the following subsection, we use Hermes to simulate filaments in the view of the Gas Puff Imaging diagnostic for W7-X.

\subsection{Filament propagation in the GPI view}

To aid in future experimental comparison, initial conditions have been motivated by the current, but limited understanding of filaments as measured in W7-X (Killer et al. 2020). Namely, filaments have been initialized as a $30 \%$ density perturbation above a background $\left(n_{0}=2 e 19 \mathrm{~m}^{-3}\right)$ and a background electron temperature of $35 \mathrm{eV}$ - with no initial temperature perturbation. The simulation domain is shown in figure 10 and has a resolution of approximately $0.5 \rho_{s}$.

Referring to (2.3), one can see that for the SOL region as viewed by the GPI diagnostic should expect filaments which are approximately $1 \mathrm{~cm}$ in diameter. However, this equation assumes that filaments will exhibit a circular cross-section in the drift plane. A filament in W7-X which exhibits a circular cross-section at one toroidal location will be significantly distorted at other toroidal locations. For this reason, we assumed that a filament is circular at the bean-shaped cross-section, and therefore slightly elliptical at the GPI view plane. While several studies have investigated the effect of ellipticity on filament propagation (e.g. Omotani et al. 2016), these have been done only in tokamak or slab geometries. The propagation of a filament in a full stellarator geometry, including distortion due to the non-axisymmetric field, is outside the scope of this work. It was determined in Killer et al. (2020), however, that filament ellipticity in a drift-plane geometry altered filament velocity by $\pm 20 \%$. These errors would not alter the main conclusions of the simulations presented here.

The radial electric field in the SOL of W7-X has been included as a constant $5 \mathrm{kV} \mathrm{m}^{-1}$ (electron-root) background electric field as motivated by probe and reflectometry measurements (Windisch et al. 2017; Killer et al. 2019). This induces a $2 \mathrm{~km} \mathrm{~s}^{-1}$ poloidal motion of the filament. In comparison, a filament with the initial 


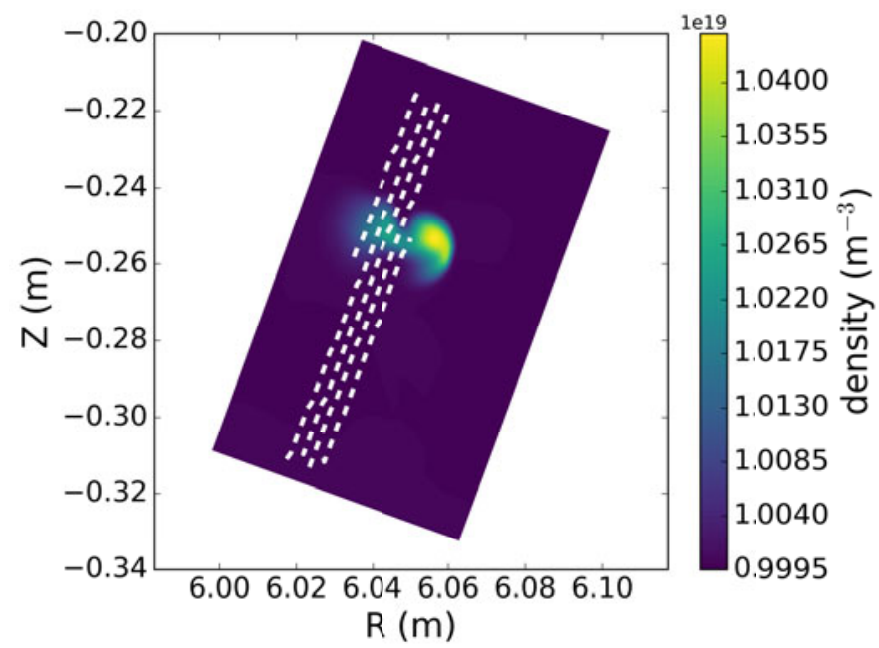

FIGURE 11. Simulated trajectory of a single seeded filament as viewed by the GPI diagnostic in W7-X. The trajectory of the filament (white, dashed) is primarily poloidal, since the radial electric field is much larger than the electric field generated by diamagnetic drifts within the filament.

conditions described here will exhibit a maximum radial velocity around $300 \mathrm{~m} \mathrm{~s}^{-1}$. As a result, the filament exhibits a primarily poloidal trajectory, as shown in figure 11 . These simulations use a periodic domain, as otherwise the filament would almost immediately leave the field of view. This assumption is motivated by the alignment of the GPI view with the last closed flux surface (LCFS); it is assumed that filaments will be generated in a somewhat poloidally uniform nature. Furthermore, there are no abrupt changes in $L_{\|}$when traversing poloidally along the LCFS.

A second acceleration is seen once the filament enters the closed-field-line region of the magnetic island, however, the low radial velocity of the filament dictates that the filament lifetime must exceed $100 \mu \mathrm{s}$. This is unlikely, as filaments observed in the SOL of W7-X had an estimated lifetime of only a few $10 \mathrm{~s}$ of microseconds (Killer et al. 2020). Therefore, only filaments which are generated near the island O-point region could experience an abrupt change in parallel connection length, and thereby a significant radial acceleration. However, the radial electric field profile and poloidal dynamics of this region are still a matter of active research, and any assertions of filament dynamics in this complex topology would be speculation. It can be concluded, however, that most filaments similar to the ones simulated here will only contribute to local transport, as their radial propagation is slow.

\section{Discussion}

The island divertor topology of the Wendelstein 7-X stellarator exhibits highly varying regions of connection length to the sheath. Parallel currents to the sheath determine filament propagation, and therefore these transitions can dictate the behaviour of filaments in the W7-X SOL. This work details simulations of plasma filaments in drift-plane geometries with abrupt transitions in parallel connection length. It is determined that a filament will be decelerated when entering a region of shorter connection length, and accelerated upon encountering a region of longer connection 
length. This effect is more prominent in filaments with a larger diameter in the drift plane, as the parallel dynamics is more prominent in this sheath-limited regime. It is also determined that a filament whose dipole develops in two separate areas of parallel connection length will be steered into the regions of higher connection length, as currents cannot dissipate the charge in this region. It is also determined that a small area of varied connection length can alter the trajectory of filaments - but the thickness of this area in the binormal direction determines the overall effect on blob propagation. A filament encountering a small region of increased parallel connection length will be accelerated, but a wide area of long connection length will ultimately lead to a deceleration at the location of the second transition. Even a small area of short connection length can drastically decelerate the filament, as parallel currents can quickly dissipate the potential dipole.

This work was then applied to experimental scenarios expected in the second operation phase of Wendelstein 7-X. Namely, filaments were simulated in drift-plane geometries which mimicked the view of the GPI diagnostic. It was determined that filaments exhibit a slow radial propagation relative to the poloidal propagation caused by the radial electric field. This leads to a predominantly poloidal motion as viewed by the GPI diagnostic.

It is worth discussing the radial velocity of the filaments simulated here with W7-X-relevant parameters. Most notably, filaments shown here are much slower than those in other plasma physics experiments - even those with comparable SOL temperatures and densities. However, one must consider the mechanisms at play. A filament dipole - and therefore the electric field which drives motion - is created due to diamagnetic drifts (curvature and $\nabla B$ ). Acknowledging previous work (Shanahan et al. 2018) which indicated that this curvature drive could be approximated as an average curvature, we see that the large aspect ratio of Wendelstein 7-X leads to a small polarizing force. This effect is somewhat mitigated by the large connection lengths of the W7-X island divertor topology. Long connection lengths inhibit charge dissipation through the sheath, increasing filament velocity. Therefore there should be some moderate radial velocity apparent, which was not the case in Wendelstein 7-AS (Hirsch et al. 2008), where filaments exhibited a negligible radial velocity in the plasma frame.

The slow radial motion of filaments in Wendelstein 7-X, as seen both here and in Killer et al. (2020), indicates that filamentary transport is radially localized - that is, filaments do not stray far from the radial location of their generation. Therefore, the observance of filaments entering a drastically new regime of $L_{\|}$in the W7-X SOL will be an uncommon occurrence. Furthermore, the strong poloidal propagation of filaments will make it difficult to disentangle to radial and poloidal motion - as it is only the radial velocity that is affected by the change in $L_{\|}$. Nevertheless, should a filament abruptly enter a new regime of $L_{\|}$, one could observe the effects demonstrated in $\S 2$.

Finally, we can derive an approximate radial diffusion coefficient for these filaments. Considering their radial velocity of around $v=300 \mathrm{~m} \mathrm{~s}^{-1}$, and using a typical length scale $\ell=3 \mathrm{~cm}$, we can deduce from $D_{\perp}=v_{D} \ell$ a diffusion coefficient $D_{\perp}$ of order unity. This is the similar to the value that is often prescribed for anomalous diffusion in W7-X transport simulations (Effenberg et al. 2017, 2019). Filaments like the ones shown here will not significantly alter the assumptions for anomalous radial transport in $\mathrm{W} 7-\mathrm{X}$. 


\section{Acknowledgements}

The authors would like to acknowledge the work of the BOUT++ development team. BOUT++ is an open-source framework available at boutproject.github.io. The model and input files used in this work are available at github.com/bshanahan. The primary author (B.S.) would also like to thank B. Dudson, A. von Stechow and C. Killer for many useful discussions. This work has been carried out within the framework of the EUROfusion Consortium and has received funding from the Euratom research and training programme 2014-2018 and 2019-2020 under grant agreement no. 633053. The views and opinions expressed herein do not necessarily reflect those of the European Commission.

\section{REFERENCES}

Angus, J. R. \& Umansky, M. V. 2014 Modeling of large amplitude plasma blobs in threedimensions. Phys. Plasmas 21 (1), 012514.

AYdemiR, A. Y. 2005 Convective transport in the scrape-off layer of tokamaks. Phys. Plasmas 12 (6), 062503.

Balay, S., Gropp, W. D., McInnes, L. C. \& Smith, B. F. 1997 Efficient management of parallelism in object-oriented numerical software libraries. In Modern Software Tools for Scientific Computing, pp. 163-202. Springer.

Benkadda, S., Garbet, X. \& Verga, A. 1994 Interchange instability turbulence model in edge tokamak plasma. Contrib. Plasma Phys. 34 (2-3), 247-252.

D'Ippolito, D. A., Myra, J. R. \& Zweben, S. J. 2011 Convective transport by intermittent blob-filaments: comparison of theory and experiment. Phys. Plasmas 18 (6), 060501.

D’Ippolito, D. A., Myra, J. R., Krasheninnikov, S. I., Yu, G. \& Pigarov, A. Y. 2004 Blob transport in the tokamak scrape-off-layer. Contrib. Plasma Phys. 44 (1-3), 205-216.

Dudson, B., Hill, P., Dickinson, D., Parker, J., Allen, A., Breyiannia, G., Brown, J., EASY, L., FARley, S., Friedman, B. et al. 2019. Bout++ v4.2.2.

Dudson, B. D. \& Leddy, J. 2017 Hermes: global plasma edge fluid turbulence simulations. Plasma Phys. Control. Fusion 59 (5), 054010.

Dudson, B. D., Madsen, J., Omotani, J., Hill, P., Easy, L. \& Løiten, M. 2016 Verification of BOUT++ by the method of manufactured solutions. Phys. Plasmas 23 (6), 062303.

Dudson, B. D., Umansky, M. V., Xu, X. Q., Snyder, P. B. \& Wilson, H. R. 2009 BOUT++: A framework for parallel plasma fluid simulations. Comput. Phys. Commun. 180, 1467-1480.

D'IPpolito, D. A. \& MYRA, J. R. 2003 Blob stability and transport in the scrape-off-layer. Phys. Plasmas 10 (10), 4029-4039.

D'Ippolito, D. A., Myra, J. R. \& Krasheninnikov, S. I. 2002 Cross-field blob transport in tokamak scrape-off-layer plasmas. Phys. Plasmas 9 (1), 222-233.

Easy, L., Militello, F., Omotani, J., Dudson, B., HavlíčKová, E., Tamain, P., Naulin, V. \& NiELSEN, A. H. 2014 Three dimensional simulations of plasma filaments in the scrape off layer: a comparison with models of reduced dimensionality. Phys. Plasmas 21 (12), 122515.

Editors, I. P. B., an Co-Chairs, I. P. E. G. C., Team, I. J. C. \& Unit, P. 1999 Chapter 1: overview and summary. Nucl. Fusion 39 (12), 2137-2174.

Effenberg, F., Feng, Y., Schmitz, O., Frerichs, H., Bozhenkov, S. A., Hlbe, H., Knig, R., Krychowiak, M., Pedersen, T. S., Reiter, D. et al. 2017 Numerical investigation of plasma edge transport and limiter heat fluxes in wendelstein 7-X startup plasmas with EMC3EIRENE. Nucl. Fusion 57 (3), 036021.

Effenberg, F., Niemann, H., Feng, Y., Geiger, J., Schmitz, O., Suzuki, Y., Ali, A., Barbui, T., BREZINSEK, S., Frerichs, H. et al. 2019 Investigation of 3D effects on heat fluxes in performance-optimized island divertor configurations at wendelstein 7-X. Nucl. Mater. Energy 18, 262-267.

Garbet, X., Laurent, L., Roubin, J.-P. \& Samain, A. 1991 A model for the turbulence in the scrape-off layer of tokamaks. Nucl. Fusion 31 (5), 967. 
Garcia, O. E., Bian, N. H. \& Fundamenski, W. 2006 Radial interchange motions of plasma filaments. Phys. Plasmas 13 (8), 082309.

Garcia, O. E., Naulin, V., Nielsen, A. H. \& Rasmussen, J. J. 2005 Turbulence and intermittent transport at the boundary of magnetized plasmas. Phys. Plasmas 12 (6), 062309.

Hammond, K. C., Gao, Y., Jakubowski, M., Killer, C., Niemann, H., Rudischhauser, L., Ali, A., Andreeva, T., Blackwell, B. D., Brunner, K. J. et al. 2019 Drift effects on W7-X divertor heat and particle fluxes. Plasma Phys. Control. Fusion 61 (12), 125001.

Hirsch, M., Baldzuhn, J., Beidler, C., Brakel, R., Burhenn, R., Dinklage, A., Ehmler, H., Endler, M., ERCKMAnN, V., Feng, Y. et al. 2008 Major results from the stellarator Wendelstein 7-AS. Plasma Phys. Control. Fusion 50 (5), 053001.

Killer, C., Grulke, O., Drews, P., Gao, Y., Jakubowski, M., Knieps, A., Nicolai, D., Niemann, H., Sitjes, A. P. \& Satheeswaran, G. 2019 Characterization of the W7-X scrape-off layer using reciprocating probes. Nucl. Fusion 59 (8), 086013.

Killer, C., Shanahan, B. W., Grulke, O., Endler, M. B. S., Hammond, K. \& RUdischHAuser, L. 2020 Plasma filaments in the scrape-off layer of wendelstein 7-X. Plasma Phys. Control. Fusion, doi:10.1088/1361-6587/ab9313.

Kocsis, G., Alonso, A., Biedermann, C., Cseh, G., Dinklage, A., Grulke, O., Jakubowski, M., KÖNIG, R., KRYChOWIAK, M., OTTE, M. et al. 2017 Characterisation of edge filamentary structures in the 3D geometry of wendelstein 7-X limiter plasmas. In 44th EPS Conference on Plasma Physics.

Krasheninnikov, S., RYutov, D. \& YU, G. 2004 Large plasma pressure perturbations and radial convective transport in a tokamak. J. Plasma Fusion Res. 6 (UCRL-JRNL-202203).

Krasheninnikov, S. I. 2001 On scrape off layer plasma transport. Phys. Lett. A 283 (5), 368-370.

KubE, R. \& GARCiA, O. E. 2011 Velocity scaling for filament motion in scrape-off layer plasmas. Phys. Plasmas 18 (10), 102314.

Madsen, J. 2013 Full-f gyrofluid model. Phys. Plasmas 20, 072301.

Myra, J. R., D’Ippolito, D. A., Krasheninnikov, S. I. \& YU, G. Q. 2004 Convective transport in the scrape-off-layer by nonthermalized spinning blobs. Phys. Plasmas 11 (9), 4267-4274.

Myra, J. R., Russell, D. A. \& D'Ippolito, D. A. 2006 Collisionality and magnetic geometry effects on tokamak edge turbulent transport. I. A two-region model with application to blobs. Phys. Plasmas 13 (11).

Omotani, J. T., Militello, F., Easy, L. \& Walkden, N. R. 2016 The effects of shape and amplitude on the velocity of scrape-off layer filaments. Plasma Phys. Control. Fusion 58 (1), 014030.

RIVA, F. et al. 2016 Blob dynamics in the TORPEX experiment: a multi-code validation. Plasma Phys. Control. Fusion 58 (4), 044005.

Schwörer, D., Walkden, N. R., Leggate, H., Dudson, B. D., Militello, F., Downes, T. \& TURNER, M. M. 2018 Influence of plasma background on 3D scrape-off layer filaments. Plasma Phys. Control. Fusion 61 (2), 025008.

Shanahan, B., Dudson, B. \& Hill, P. 2018 The effects of non-uniform drive on plasma filaments. J. Phys.: Conf. Ser. 1125, 012018.

Shanahan, B., Dudson, B.\& Hill, P. 2019 Fluid simulations of plasma filaments in stellarator geometries with BSTING. Plasma Phys. Control. Fusion 61 (2), 025007.

Shanahan, B. W. \& Dudson, B. D. 2016 Blob dynamics in torpex poloidal null configurations. Plasma Phys. Control. Fusion 58 (12), 125003.

Walkden, N. R., Dudson, B. D. \& Fishpool, G. 2013 Characterization of 3D filament dynamics in a mast SOL flux tube geometry. Plasma Phys. Control. Fusion 55 (10), 105005.

Walkden, N. R., Easy, L., Militello, F. \& Omotani, J. T. 2016 Dynamics of 3D isolated thermal filaments. Plasma Phys. Control. Fusion 58 (11), 115010.

Wiesenberger, M., Madsen, J.\& Kendl, A. 2014 Radial convection of finite ion temperature, high amplitude plasma blobs. Phys. Plasmas 21 (9), 092301.

Windisch, T., Krmer-Flecken, A., Velasco, J. L., Knies, A., Nhrenberg, C., Grulke, O. \& KLINGer, T. 2017 Poloidal correlation reflectometry at W7-X: radial electric field and coherent fluctuations. Plasma Phys. Control. Fusion 59 (10), 105002. 
YU, G. Q. \& KRasheninnikov, S. I. 2003 Dynamics of blobs in scrape-off-layer/shadow regions of tokamaks and linear devices. Phys. Plasmas 10 (11), 4413-4418.

Zoletnik, S., Anda, G., Biedermann, C., Carralero, A. D., Cseh, G., Dunai, D., Killer, C., Kocsis, G., Krmer-Flecken, A., Otte, M. et al. 2019 Multi-diagnostic analysis of plasma filaments in the island divertor. Plasma Phys. Control. Fusion 62 (1), 014017. 